\title{
Multilinguales
}

1 | 2013

Pratiques littéraires, linguistiques, pédagogiques,

didactiques et médiations culturelles contemporaines

\section{L'école française dans le fils du pauvre de Mouloud Feraoun : Rite de passage ou rite d'institution?}

The French School in "The Son of the poor man" of Mouloud Feraoun: Rite of Passage or Rite of Institution?

\section{Farida Boualit}

\section{OpenEdition}

\section{Journals}

Édition électronique

URL : https://journals.openedition.org/multilinguales/2904

DOI : $10.4000 /$ multilinguales.2904

ISSN : 2335-1853

\section{Éditeur}

Université Abderrahmane Mira - Bejaia

\section{Édition imprimée}

Date de publication : 1 juin 2013

Pagination : $31-43$

ISSN : 2335-1535

Référence électronique

Farida Boualit, «L'école française dans le fils du pauvre de Mouloud Feraoun : Rite de passage ou rite d'institution? », Multilinguales [En ligne], 1 | 2013, mis en ligne le 01 juin 2013, consulté le 30 juin 2021. URL : http://journals.openedition.org/multilinguales/2904 ; DOI : https://doi.org/10.4000/ multilinguales. 2904

Ce document a été généré automatiquement le 30 juin 2021.

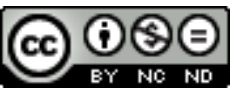

Multilinguales est mise à disposition selon les termes de la Licence Creative Commons Attribution Pas d'Utilisation Commerciale - Pas de Modification 4.0 International 


\title{
L'école française dans le fils $d u$ pauvre de Mouloud Feraoun : Rite de passage ou rite d'institution?
}

\author{
The French School in "The Son of the poor man" of Mouloud Feraoun: Rite of \\ Passage or Rite of Institution?
}

Farida Boualit

1 Depuis près d'un demi-siècle, les travaux de recherche sur les œuvres de Mouloud Feraoun, dans leur majorité, jusqu'à ceux du dernier colloque international organisé en son hommage (Alger, mars 2012), reviennent à vouloir répondre à une seule et même question : son projet d'écriture s'inscrit-il dans l'idéologie coloniale? Autrement dit, en tant que colonisé ayant fréquenté l'école française, était-il assimilationniste? Les critiques convaincus qu'il ne l'était pas en sont encore à demander à ceux qui le sont de «relire » son œuvre pour s'en persuader. Le paradoxe est que nous arguons tous de la représentation de la culture dans ses textes pour le prouver. Le fort ancrage des œuvres de Feraoun dans l'habitus socius kabyle serait, pour les uns, une manière d'affirmer l'identité autochtone niée par le colonisateur, et pour les autres, une autoreprésentation ethnographique (exotique) qui répondrait à l'attente de ce même colonisateur.

2 Nous avons donc opté pour l'analyse de cet enjeu qu'est la culture des textes de Mouloud Feraoun, en nous référant au Fils du pauvre parce que dans ce roman, la question de la culture se "complexifie » avec l'intrusion de l'école française dans l'univers autochtone. Notre objectif est de démontrer que sa «thématisation », dans le contexte de la société kabyle au temps de la colonisation, est ambivalente. Du point de vue de sa représentation en tant que fait culturel, l'école (française) est bien décrite comme un « rite de passage » (en soi), selon la conception d'A. Van Gennep ${ }^{1}$.

3 Mais ce rite, tel que vécu par l'initié Fouroulou, en chacune de ses trois étapes, n'est pas accompli dans sa plénitude, comme nous le verrons ici même. Pour saisir le sens de ce comportement en retrait, nous avons dû convoquer la notion de « rite d'institution » 
que P. Bourdieu² préfère à celle de « rite de passage » car, selon lui, l'acte d'institution est un acte de communication, mais d'une espèce particulière: il signifie à quelqu'un son identité, mais au sens à la fois où il la lui exprime et la lui impose en l'exprimant à la face de tous. (59)

4 Dans le cas de Fouroulou, cette identité, «signifiée/imposée " par le rituel scolaire, est étrangère à sa communauté, et implique, si elle est assumée, «l'institution » d'une différence radicale entre lui et les siens. Selon P. Bourdieu,

en marquant solennellement le passage d'une ligne qui instaure une division fondamentale de l'ordre social, le rite attire l'attention de l'observateur vers le passage (d'où l'expression rite de passage), alors que l'important est la ligne. Cette ligne, en effet, que sépare-t-elle ? Un avant et un après, bien sûr (...). (58)

5 Tout le roman du Fils du pauvre est travaillé par la (vaine) négociation dans le but de neutraliser « la ligne » entre cet « avant » et cet « après ».

\section{Question de méthode}

6 Pour cerner un tel objectif, la démarche ethnocritique nous a paru tout à fait appropriée. Cette discipline, qui s'appuie sur l'anthropologie, ouvre des perspectives nouvelles à la lecture des textes littéraires surdéterminés par leurs relations à la culture d'une société ou d'un groupe social, comme c'est le cas pour les textes de Mouloud Feraoun. Cependant, elle se propose d'étudier « la culture du texte» et non «la culture dans le texte $»^{3}$.

7 Or, jusqu'à présent, la relation de l'anthropologie et de la littérature, du moins pour ce qui concerne la littérature maghrébine, consistait à se référer à la première pour détecter la présence d'éléments culturels dans la seconde. C'est cette posture méthodologique qui explique que les œuvres de Feraoun, en l'occurrence, aient été lues comme le «document-témoignage »d'un (instituteur) Kabyle sur le quotidien des Kabyles, à l'intention des Français/Européens, et classées dans «le courant ethnographique».

8 Pour comprendre l'enjeu d'un tel classement, il faut revenir à la conception de l'anthropologie dans les années cinquante. Avant qu'elle n'évolue, surtout depuis les indépendances des ex-colonies, l'anthropologie se confondait avec l'ethnographie et était déterminée par son " occidentalité ». De ce fait, elle se cantonnait à l'observation et à la description des mœurs et coutumes des ethnies « exotiques ", " primitives ".

C'est en référence à un tel contexte que certaines œuvres algériennes francophones (mais pas seulement), de la même époque, ont été qualifiées d'« ethnographiques » et identifiées au courant du même nom: elles décriraient les us et coutumes des Algériens, mais pour l'étranger européen en le confortant dans sa vision ethnocentriste. En guise d'illustration littéraire prototypique de ce courant, la critique citait et cite toujours l'œuvre de Mouloud Feraoun, notamment Le Fils du pauvre. Grâce aux outils de l'ethnocritique, nous pensons pouvoir dépasser le repérage réducteur de «la culture dans le texte», pour atteindre «la culture du texte» de Feraoun. Pour mettre en relief l'apport incontestable de cette discipline à la lecture des corpus dont les auteurs se présentent ou sont présentés comme des "écrivains-ethnographes ", à l'instar de Mouloud Feraoun, il nous faut revenir à ses postulats de base tels qu'énoncés par ses fondateurs. 
10 Le principe le plus important, dans le cas de notre étude, est celui de la textualisation de la culture. Il est exploité par Marie Scarpa dans son ouvrage Le Carnaval des Halles, Etude sociocritique du Ventre de Paris de Zola (CNRS Editions, 2000) dans un sens qui s'applique parfaitement à l'œuvre de Mouloud Feraoun :

Il va sans dire que l'ethnocritique ne se contente pas du simple repérage des faits ethnographiques, des « documents » et autres items folkloriques (...) présents dans le texte littéraire (...). Bref, elle souhaite échapper à la déculturation comme à la détextualisation de l'œuvre étudiée (...). L'ethnocritique se propose donc de lire les "documents" dans leur valeur ethnographique, au ras du texte, puis de voir comment ils construisent ce qu'on peut appeler l'ethno-poétique de l'œuvre. (11-13)

11 C'est précisément l'objectif que nous voulons atteindre avec le texte de Feraoun qui ne cesse de poser, à l'instar du texte de Zola, la question du document. Certes, Mouloud Feraoun, contrairement à Emile Zola, ne s'est pas identifié lui-même à un «écrivainethnographe », mais des critiques, comme A. Khatibi, J. Dejeux et bien d'autres, l'ont fait pour lui.

12 Le second principe est celui de l'objet d'étude de l'ethnocritique tel que défini par JeanMarie Privat et Marie Scarpa qui précisent, dans la présentation de l'ouvrage collectif Horizons ethnocritiques (P.U. Nancy, 2010), qu'« elle analyse en somme la dialogie culturelle à l'œuvre dans les textes littéraires»(8), parce qu'elle "présuppose qu'il y a non seulement diffraction "du même chez l'autre" mais aussi dissémination " de l'autre dans le même" » (8).

Pour estimer le bénéfice que nous escomptons de la définition de l'objet d'étude de l'ethnocritique, il nous faut rappeler l'hypothèse qui le porte. Sa formulation dans l'article Pour une lecture ethnocritique de la littérature par Marie Scarpa ${ }^{4}$, se référant à Jean-Marie Privat, en offre une synthèse des plus opératoires :

Elle (l'ethnocritique) s'intéresse donc fondamentalement à la polyphonie culturelle et plus spécialement, pour l'instant en tout cas, aux formes de culture dominée, populaire, folklorique, illégitime dans la littérature écrite dominante, savante, cultivée, légitime. Les œuvres apparaissent dès lors comme des "bricolages" (ou "bris-collages") culturels, configurés selon des processus spécifiques : une lecture ethnocritique s'attache à rendre compte de ce dialogisme culturel, et de sa dynamique, plus ou moins conflictuelle. (1)

Cet intérêt de l'ethnocritique pour les "formes de culture dominée, populaire, ...», dans des œuvres assimilées à des "bricolages culturels", travaillées par une "dynamique conflictuelle» dont elle veut rendre compte, constitue le troisième principe et correspond tout à fait à ce que nous voulons cibler du Fils du pauvre. Au terme de la mise en œuvre de ces trois paramètres fondamentaux de l'ethnocritique, nous aurons tenté de contribuer à l'élaboration d'une ethno-poétique de l'œuvre de Mouloud Feraoun qui ouvre le texte à sa culture (" culture du texte »), tout en évitant sa " détextualisation » (« culture dans le texte »).

\section{La dialogie culturelle dans Le fils du pauvre : le déplacement des frontières}

15 Pour analyser la « culture du texte » féraounien, nous souhaitons reprendre le débat là où l'ont laissé Martine Mathieu-Job et Robert Elbaz, dans Mouloud Feraoun ou l'émergence d'une littérature (Karthala, 2001). Pour ces deux chercheurs Le Fils du pauvre est «le roman fondateur de la littérature maghrébine" (11) qu'ils définissent comme "une 
littérature originale se démarquant des écritures exotiques et a fortiori des écritures coloniales produites par des écrivains français qui s'étaient saisis jusque-là du référent maghrébin » (5).

Ils commencent par poser que cet écrivain est porteur d'un projet d'écriture résolument novateur qu'il partage avec «ceux qui ont rompu avec un Orient de pacotille pour décrire une humanité moins belle et plus vraie", selon les propres déclarations de l'auteur ${ }^{5}$. C'est ce que vont démontrer ces deux auteurs en recentrant leurs recherches sur le texte, tout en se démarquant d'une certaine critique française du texte féraounien marquée, selon eux, par «le primat du référent sur le travail de l'écriture » (7). Nous pouvons ajouter qu'il en est de même pour la critique algérienne dans sa majorité. Ils reconnaissent que le texte de Feraoun est caractérisé par la coprésence de deux types d'énoncés, narratifs et socio-anthropologiques, dont ils vont évaluer l'enjeu :

Dans l'option de Feraoun, il se fait un équilibre véritable entre ces énoncés socioanthropologiques qui parsèment le roman dans son entier et les énoncés narratifs qui nous racontent l'histoire de Menrad : c'est à cette condition que se réalise la cohésion du roman, nonobstant l'amputation du texte par les éditeurs français, et qu'elle se maintient du début à la fin, malgré l'éparpillement du texte. (25)

Nous tentons, pour notre part, de démontrer que cette "cohésion du roman", qui subsume la coprésence des énoncés narratifs et des énoncés socio-anthropologiques, est aussi l'effet de la dimension anthropologique de certains "énoncés narratifs qui racontent l'histoire de Menrad ", comme celui de l'école française.

Certes, la «culture kabyle dans le texte féraounien » est fortement mise en scène. Mais sa textualisation efface en elle tout indice d'exotisme. Nous pourrions démontrer, en effet, qu'aucune coutume n'est décrite comme un spectacle pittoresque offert au regard d'un lecteur français/européen. Bien au contraire, il y a chez Mouloud Feraoun un parti pris de banalisation de tout ce qui concerne la culture kabyle et ce, depuis l'incipit du Fils du pauvre:

Le touriste qui ose pénétrer au cœur de la Kabylie admire par conviction ou par devoir des sites qu'il trouve merveilleux (...). On peut le croire sans difficulté du moment qu'il retrouve n'importe où les mêmes merveilles (...). (...) Mille pardons à tous les touristes. C'est parce que vous passez en touristes que vous découvrez ces merveilles et cette poésie. Votre rêve se termine à votre retour chez vous et la banalité vous attend sur le seuil. Nous, Kabyles, nous comprenons qu'on loue notre pays. (8)

19 La récusation du touriste, c'est-à-dire du prisme culturel exotique, prend place dans un programme d'écriture de la banalisation de la vie kabyle que nous avons étudié dans un article intitulé « » l'écriture de l'insinuation et du trompe-l'œil de Mouloud Féraoun »6. Dans cette publication, nous avons tenté de démontrer que les particularités anthropologiques kabyles ne s'affirmaient pas en tant que telles dans les textes de Mouloud Feraoun. Quand elles ne sont pas déniées aussitôt que décrites (comme l'organisation en karoubas dans La Terre et le sang, Seuil, 1953), elles sont insérées dans la diégèse, avec une forte dimension fictionnelle. Pour emporter la conviction, nous ne prendrons ici qu'un seul exemple (extrême), extrait de la partie dont Le Fils du pauvre a été délesté : "Il (Fouroulou) se maria exactement comme on se marie chez lui, ni mieux ni plus mal. Inutile de le raconter. Beaucoup connaissent cette cérémonie. Les autres ne perdent pas grand-chose à l'ignorer " (L'Anniversaire : p. 131).

20 Ce type d'ellipses "descriptives ", surdéterminées par leur dimension métadiscursive, et dont nous pourrions multiplier les exemples, s'ajoute aux procédés de banalisation des us et coutumes des Kabyles pour conforter le projet de Mouloud Feraoun de rompre 
avec « un Orient de pacotille». Nous étions parvenue à la conclusion que, dans les romans de Feraoun, c'est le narratif (la fiction) qui gère le descriptif (socio-anthropologique) et non l'inverse, comme le laissent penser ceux qui traitent ses textes comme des documents (ethnographiques).

Dans la présente étude, nous allons mettre au jour la dimension anthropologique d'un énoncé narratif clé, la fréquentation de l'école française par Fouroulou Menrad. Le choix de ce signifié narratif est d'autant plus pertinent pour notre démonstration que les critiques identifient généralement Mouloud Feraoun à l'école coloniale française et Le Fils du pauvre à l'emblème de cette adhésion?

Dans notre publication, citée plus haut, nous avons proposé une lecture autre de la présence des énoncés scolaires dans le texte de Feraoun. En nous référant à la pratique de détournement de la "la perruque», telle que définie par Michel de Certeau dans Invention du quotidien (Tome1, 1990 : p. 8), nous avons analysé les modalités d'écriture de deux types de perruques dont Feraoun use pour sinsinue ( $r$ ) dans le système culturel colonial et y inscrire sa présence et celle des siens : la perruque littéraire et la perruque scolaire.

Nous en avons déduit que l'identification, dans le texte (même autobiographique), d'énoncés scolaires de manuels utilisés à l'école française, à l'époque de Feraoun, ne permettait pas, à elle seule, de conclure à la reconnaissance pure et simple du système colonial par le "personnage/écrivain ». La dialogie culturelle qui travaille le texte est celle de «l'autre » qui pose son identité différentielle sur le terrain du même. Cette dialogie est conflictuelle dans la mesure où le déplacement sur ce terrain " étranger " implique une égalité entre l'autre et le même qui, lui, cultive la distance :

Menrad est kabyle. Ce n'est pas de sa faute. Il connaît les Français depuis son jeune âge. Les premiers furent les gendarmes. (...) Ils (les Français des petites villes) méprisent l'indigène, ils veulent à toute force former une caste à part et ne pas voir les autres. Fouroulou, encore jeune s'est aperçu de ces choses. (...) Ses professeurs, eux-mêmes, favorisaient ses camarades français et certains internes. Il se vit obligé d'être inférieur et détestable. Il se résigna. (L'Anniversaire : pp. 115 -116)

Ainsi, les Français et l'école française (tous paliers confondus) renvoient à Fouroulou Menrad une image dégradée de lui-même, dont il est conscient, et dont il faut tenir compte pour comprendre la position du personnage/écrivain par rapport aux réalités culturelles en présence. C'est ce que nous comptons faire ici, en nous intéressant, cette fois, à l'école française dans Le Fils du pauvre.

\section{Fouroulou et l'école française : un rite de passage, mais...}

25 A travers le parcours scolaire de Fouroulou Menrad, il est donné à comprendre que la fréquentation de l'école française est bien un rite de passage tel que défini par A. Van Gennep, mais que celui-ci reste inaccompli: Fouroulou Menrad n'accédera pas pleinement au stade final du rite, à savoir l'agrégation achevée à un monde nouveau, avec un statut nouveau. Fouroulou aura bien, après l'Ecole normale, un nouveau statut, mais dans le monde ancien (celui d'avant l'initiation) dont il ne s'est jamais séparé.

26 L'accès à l'école française de Fouroulou et de l'auteur, puisque l'œuvre est autobiographique, est présenté comme l'opportunité d'une promotion socioéconomique : échapper à un destin tout tracé de berger. Ce changement de statut, à la 
faveur du passage par l'école française, a toujours été l'argument d'une certaine critique pour étayer la thèse de l'assimilation de Mouloud Feraoun à la culture française et de son acculturation.

Or, notre hypothèse est que l'école française est bien conçue comme un rite de passage, mais l'intéressé se tenant toujours en retrait de son initiation, on ne peut pas conclure à son intégration. Pour le démontrer, nous allons exploiter la définition du rite de passage de A. Van Gennep (cité plus haut) et les deux aspects du rite mis en valeur par Martine Segalen dans Rites et rituels contemporains (1998). Le premier est la composition du rite :

Le rite ou rituel est un ensemble d'actes formalisés, expressifs, porteurs d'une dimension symbolique. Il est caractérisé par une configuration spatio-temporelle spécifique, par le recours à une série d'objets, par des systèmes de comportements et de langages spécifiques, par des signes emblématiques dont le sens codé constitue l'un des biens communs d'un groupe. (20)

A cette définition du rite, s'ajoute le second aspect, qui est la condition sine qua non du rite, à savoir qu'il «se situe dans l'acte de croire à son effet, à travers des pratiques de symbolisation» (17).

Si tel est le rite de passage, c'est-à-dire un « ensemble d'actes formalisés » qui « se situe(nt) dans l'acte de croire à son effet », l'école française devrait signer l'adhésion "fervente » de Fouroulou (et de Feraoun) au rituel scolaire. Certes, le parcours scolaire avec ses différents paliers (certificats d'études, brevet, concours) est bien un « ensemble d'actes formalisés ", mais ceux-ci ne sont pas "porteurs d'une dimension symbolique», pas même l'étape inaugurale du rite, «l'entrée à l'école » de Fouroulou :

Je me souviens, comme si cela datait d'hier, de mon entrée à l'école. (...) Je fus débarbouillé en hâte et cinq minutes après, encore abasourdi, je débarquai dans la vaste cour de l'école, toute grouillante d'élèves...à cent lieues de mon déjeuner. (...) Mon camarade Akli, qui était resté mon protecteur, m’avait devancé d'un an dans cette nouvelle situation. Tous les matins, il m'appelait, il m'attendait, il me ramenait. $(48 / 49 / 50)$

La rentrée est bien une "séparation", étape initiale du rite de passage. Cette phase "préliminaire" n'est pourtant pas radicale en ce que le lien de Fouroulou avec l'univers initial est maintenu à travers un rituel de liaison : « tous les matins, il m'appelait, il m'attendait, il me ramenait ». D'autres exemples peuvent en attester, comme celui-ci : "Chaque année, aux grandes vacances, il (Fouroulou) revenait parmi les siens » (125).

31 Nous pouvons considérer que la phase de « séparation » n'est pas accomplie parce qu'il n'y a pas à proprement parler de scission avec la communauté : l'étape préliminaire du rite est escamotée et l'accès à l'école française ne se fait pas dans la rupture avec le groupe social d'origine.

Pourtant, la différence entre les deux univers est nettement posée : tout le chapitre VI, qui précède celui de l'entrée à l'école française (chapitre VII), est consacré aux activités artisanales (poterie, tissage,...) des deux tantes de Fouroulou, et surtout au talent de conteuse de l'une d'elles: "J'assistais au triomphe du petit Mquidech qui a vaincu l'Ogresse » (46). Cette initiation de Fouroulou à la tradition orale aura des effets pardelà le temps de l'enfance : " Je suis reconnaissant à Khalti de m'avoir appris de bonne heure à rêver, à aimer créer pour moi-même un monde à ma convenance, un pays de chimères où je suis seul à pouvoir pénétrer » (47). 
33 La proximité des deux chapitres (VI-VII) suggère une relation de contiguïté, et non d'opposition, entre l'univers de la tradition orale attachée à l'enfance et l'univers de l'école française.

Il en est de même de la seconde phase dite "liminaire»: l'initiation aux «actes formalisés » qui caractérisent l'école n'a pas marqué Fouroulou : « Ma première journée de classe, ma première semaine, et même ma première année ont laissé dans ma mémoire très peu de traces. J'ai beau fouiller parmi mes souvenirs, je ne trouve rien de clair » (49).

Pourtant, il reconnaît, en aval, que « les souvenirs d'enfance manquent de précision et de lien : (mais) on garde certaines images frappantes que le cœur peut toujours unir l'une à l'autre quand il les évoque » (74). On en déduit que la rentrée à l'école française ne fait pas partie de ces «images frappantes» dont le cœur se charge de pallier les lacunes.

36 L'effacement des souvenirs de l'école, qui les soustrait à la narration, est accentué par leur récit sous forme de sommaires au sens que Gérard Genette donne à cette catégorie narrative (Discours du Récit, Seuil, 2007 : p. 92). Ce procédé narratif du sommaire est amplement utilisé pour tous les événements touchant au passage d'un palier scolaire à un autre : par exemple, les quatre années de collège sont narrées en une page (p.121). Le même traitement est réservé à l'école primaire. A la première année de classe, dont le lecteur ne saura pas grand-chose, s'ajoute le récit plus que sommaire de la deuxième année : «Ah! dit mon père, je ne m'étonne plus que ton maître se plaigne de toi. C'était en effet ma deuxième année d'école et j'étais toujours dans le même cours » (p. 51). Cette information est délivrée avec une indifférence préparée en amont: « Je serais embarrassé de dire si je fus bon ou mauvais élève, si j'appris beaucoup ou peu » (48).

De plus, ce «redoublement " n'est pas identifié à une situation d'échec scolaire, qui aurait "dramatisé » la présence de l'école dans l'univers de la fiction, puisque Fouroulou la «normalise » (la banalise) aussitôt : «A partir de ce jour, je devins bon élève presque sans effort » (52).

Ces récits sommaires des séquences scolaires, l'amnésie qui les "vide» de leur substance narrative sont les effets de l'indifférence que manifeste Fouroulou pour l'école : « Du moins, je n'éprouvai aucune répugnance à être écolier. (...) J'allais à l'école sans arrière-pensée. Simplement parce que tous les enfants y allaient» (49-50). La fréquentation de l'école n'est pas soutenue par un acte de foi sur lequel se fonde tout rite de passage. Fouroulou, par ce comportement neutre, non motivé, ne s'implique pas dans son initiation scolaire. Même la joie qu'elle pouvait lui procurer était de courte durée : «Pendant un court instant, tout au moins, Menrad fut parfaitement heureux (de sa réussite au concours d'entrée à l'Ecole normale). (...) Il eut honte de son exaltation du début, se replia sur lui-même (...) » (L'Anniversaire : p. 114).

Fouroulou n'était pas non plus «dupe»de la période de l'Ecole normale qualifiée d'« idyllique » : « Pourtant, s'il y songe bien, cette société de normaliens ne fut pas aussi idéale qu'il s'entête à le dire. Elle avait ses imperfections" (L'Anniversaire: pp.115-121). Notons que la croyance en cette "société de normaliens» se situe au niveau du «(se) dire » et non du " penser »; " s'entête à le dire » suggérant une autosuggestion qui n'atteint pas son but.

40 Les exemples qui induisent que Fouroulou n'est pas attaché à l'école française par une profession de foi essaiment dans le texte. De plus, à certains endroits, point un net 
déphasage entre l'écolier et l'univers scolaire. Des facteurs d'ordre culturel contribuent à empêcher la réalisation de "l'acte de croire " en l'école française parce que le processus de symbolisation des pratiques scolaires chez l'apprenant Fouroulou est irréductiblement entravé :

À l'âge où ses camarades s'éprenaient d'Elvire, lui, apprenait « Le Lac » seulement pour avoir une bonne note. Mais comme il débitait son texte d'un ton hargneux, au lieu d'y mettre comme il se doit la douceur mélancolique d'un cœur sensible et délicat, le professeur le gourmandait et Fouroulou allait s'asseoir plein de rancune. (125)

41 Cette incapacité de l'écolier à se conformer à un code qui lui est étranger, en dépit de sa volonté de réussir l'exercice purement scolaire de la récitation, révèle à la fois le fossé culturel que le travail même acharné de l'écolier ne peut pas combler, et la limite audelà de laquelle commence l'«intégration». Fouroulou ne la franchira à aucun moment. C'est ce qui est confirmé à la fin de la narration de l'épisode de l'Ecole normale, dans L'Anniversaire, censé participer de la seconde phase du rite de passage, la marginalisation. Fouroulou, bien qu'il ait changé de lieu, se tient à l'écart dans le lieu d'accueil : «A Alger (...). Il n'eut jamais ni aventure, ni illusion» (123). Le lieu de la marge n'est pas investi par le sujet et la fin de son initiation marque son retour au lieu d'avant la séparation : «Il (Fouroulou) sentait qu'il ne serait à l'aise que chez lui, avec des gens comme lui. Il passa ses années de ville en observateur intéressé mais non séduit, il décrocha ses diplômes et revint dans son pays sans amertume et sans colère » (123).

La troisième phase du rite, l'agrégation/le retour au pays, est bien annoncée à la suite d'une phase liminaire banalisée au niveau de son récit (sommaire) et de son absence d'impact sur l'initié dont les sentiments sont neutralisés (« sans ...et sans»).

Mais, si l'univers initial (avant la séparation) et l'univers de l'initiation (de la marge) ont coexisté à travers des rituels, si cette initiation n'est vécue ni comme une épreuve ni comme un acte de foi, peut-on encore parler de rite de passage? La réponse exige l'étude du dernier stade du rite, à savoir le devenir-instituteur de Fouroulou.

\section{Rite de passage ou rite d'institution?}

La phase de marginalisation (École normale) est exceptionnellement marquée par la coexistence avec les Français :

En dehors des affections dont elle (période de l'Ecole normale) fut remplie, mise à part son exceptionnelle fécondité intellectuelle et morale, ce qui fait encore son prix aux yeux du jeune homme, c'est que ce fut la seule qu'il vécut avec des Français: avant l'Ecole normale, il ne les connaissait guère; les trois années écoulées, il ne les voit plus que de loin. Il retourne dans son bled avec son bagage de primaire, une foule de souvenirs et un choix de belles émotions. (L'Anniversaire : p. 121)

Cette convivialité toute relative - Fouroulou Menrad évoque des divisions de classe entre normaliens qui empêchent la communication et le rapprochement - ne dure que le temps des études de Fouroulou à l'Ecole normale : elle prend fin avec le retour " dans son bled». Donc, la troisième phase, l'agrégation, n'est pas telle que la décrit A. Van Gennep. Mais, grâce à l'école française, il aura tout de même un nouveau statut au sein de sa communauté.

En effet, l'école primaire française lui a permis de s'initier à la littératie. Grâce à cette "aptitude à comprendre et à utiliser l'information écrite (en français) dans la vie 
courante 》 (OCDE, La Littératie à l'ère de l'information), Fouroulou est capable d'intercéder en faveur des siens auprès de la société (l'administration) française, en devenant une sorte d'« agent de liaison ». Il lit à la famille les lettres envoyées par le père (émigré en France) et il écrit au père pour lui donner de leurs nouvelles. Lecture et écriture se déroulent à travers un véritable rituel familial, repris à divers endroits du texte, et qui est explicitement associé au savoir-faire scolaire : " La troisième lettre qu'écrivit Fouroulou à son père commençait ainsi : "c'est avec joie que je t'écris pour t'annoncer que je suis admis au certificat...". Cette formule apprise à l'école (...) lui parut belle en elle-même et digne d'être lue à Paris» (99).

En outre, la littératie comme savoir-faire est nettement distinguée de l'écriture créatrice :

Devant les innombrables obstacles qui se dressent à chaque tournant de phrase, à chaque fin de paragraphe,(...) il abandonne une entreprise au-dessus de ses forces, après avoir rempli un gros cahier d'écolier. Il abandonne sans esprit de retour, sans colère. (6)

Contrairement à son initiation à la tradition orale, qui l'a doté du pouvoir de «créer un monde de chimères» (de fiction), Fouroulou, devenu instituteur, ne parvient pas à se servir de son lettrisme pour écrire son roman. Il se contente d'être instituteur jusqu'au jour où le narrateur qui parle de lui à la troisième personne lui donnera la parole (un autre narrateur la lui reprendra dans le dernier tiers du roman).

Rappelons également que le passage par l'école française lui a permis de gravir l'échelle sociale, du moins au début de sa carrière : «En Algérie, il était pris dans cette alternative: ou devenir instituteur, ce qui signifiait l'aisance pour toute la famille, ou redevenir berger » (126). Mais si Fouroulou est satisfait de son sort d'instituteur, son bonheur (de courte durée) est sans prétention, comme celui du «vieux Gallus» de J.M. de Heredia (Villula) :

Dans son école, "au penchant du coteau " au milieu de la verdure, avec sa petite classe, son petit jardin, sa petite famille, Fouroulou peut songer au sort du vieux Gallus qui serait bientôt le sien. (...) Il se sentait libre, aimé, respecté, heureux. C'était en 1939, le dernier printemps de la paix. (133)

50 La comparaison avec le "vieux Gallus », à qui «appartient l'héritage (...) au penchant $d u$ coteau ", qui est "satisfait de son destin borné (et qui) finit de vivre où jadis il est né», travaille la description de la vie étriquée de l'instituteur :

Rien n'est plus obscur que l'existence insignifiante du malheureux Menrad pendant ces premières années. L'horizon qui s'offre à sa vue, chaque fois qu'il se trouve dans la cour de l'école, est l'image frappante de sa propre vie. Il est restreint comme elle. (L'Anniversaire : p. 138)

Notons que, contrairement à Gallus, Fouroulou n'est pas satisfait de son sort, même sur le plan financier :

Fouroulou n'a pas bougé de son poste. (...) Il a son traitement qu'il partage avec les siens. Un petit traitement, une grande famille. (...) Ils tiennent le coup. Pourvu que cela dure, songe philosophiquement Fouroulou.. Malheureusement, cela ne dura pas. (L'Anniversaire : p. 142)

52 Feraoun est dans les mêmes dispositions que Fouroulou. Dans un article intitulé «itinéraire d'un enfant du bled ou chronique d'une mort annoncée »", nous avons tenté de montrer, en référence au Journal (ENAG, 1998) et à Lettres à ses amis (ENAG, 1998), que Feraoun n'était pas un instituteur heureux. Dans Lettres à ses amis, par exemple, il écrit : «La rentrée a été dure pour moi » (1952: p. 93) ; "Je t’assure que le métier me dégoûte (...) » 
(1958: p. 174) ; «Ici, à l'école, j'ai de plus en plus le cafard et je prends en grippe (...) le métier » (1959: p. 180); «En vérité je suis en plein dégoût!» (1959 : p. 185); «L'Académie a réussi à exprimer de moi tout ce que j'ai pu donner de bon. Il ne reste plus rien » (1959 : p. 196).

Ce sont là quelques raisons qui nous autorisent à conclure que Fouroulou/Feraoun a bien suivi le rite de passage de l'école française, mais sans s'y impliquer, sans y mettre de la «ferveur ». Le rite de passage n'a abouti ni à l'assimilation de l'initié à sa nouvelle culture, ni à son adhésion inconditionnelle à son nouveau statut.

Peut-on encore parler de rite de passage quand celui-ci n'a pas abouti sans pour autant avoir échoué ? Il nous paraît plus pertinent, dans le cas de Fouroulou/Feraoun, de l'envisager dans l'optique de P. Bourdieu qui opte pour le « rite d'institution», plus en phase avec la fonction du rite de passage dans la société :

On peut en effet se demander si, en mettant l'accent sur le passage temporel - de l'enfance à l'âge adulte par exemple-, cette théorie ne masque pas un des effets essentiels du rite, à savoir de séparer ceux qui l'ont subi non de ceux qui ne l'ont pas encore subi mais de ceux qui ne le subiront en aucune façon et instituer ainsi une différence durable entre ceux que ce rite concerne et ceux qu'il ne concerne pas. C'est pourquoi, plutôt que rites de passage, je dirais volontiers rites de consécration, ou rites de légitimation ou, tout simplement, rites d'institution (en donnant à ce mot le sens actif qu'il a par exemple dans l'expression " institution d'un héritier"). (Op. cit. p. 58)

La description que fait P. Bourdieu d'« un des effets essentiels du rite » expliquerait, de notre point de vue, la position du personnage/auteur du Fils du pauvre : l'école est bien un rite qui "institue une différence durable entre ceux que ce rite concerne et ceux qu'il ne concerne pas », au sein de la société kabyle. Ce rite " sépare » ceux qui vont (ou ont été à l'école) de ceux qui ne vont pas (ou n'iront pas) à l'école. Nous parlerons alors d'un rite de passage qui ne s'accomplit pas parce qu'il fonctionne comme un rite d'institution qui ne s'impose pas. La notion de P. Bourdieu a l'avantage de s'arrêter à la ligne pour en estimer la valeur pour celui qui a à la franchir.

C'est ce que ne fera pas Fouroulou Menrad/Mouloud Feraoun qui ne se résout pas à la séparation (radicale) avec les siens. Il n'assume pas l'institution de cette «différence durable» entre lui et les membres de sa communauté.

Pour paraphraser P. Bourdieu, nous dirons que Fouroulou/Feraoun n'a pas reconnu comme légitime, la limite que le rite scolaire a tenté d'instituer entre lui et les siens; en d'autres termes, il ne méconnaît pas comme arbitraire l'identité signifiée et imposée par ce rite.

Dès lors, comment comprendre l'identification de Feraoun à un « produit authentique de l'école française ", même si ce sont ses propres termes?

Nous avons essayé d'apporter quelques éléments de réponse en montrant que Fouroulou/Feraoun est surtout celui qui « vit au milieu des aveugles, mais qui ne veut pas se considérer comme roi» (5). Cet énoncé-incipit du Fils du pauvre programme judicieusement la lecture de la dimension du rite d'institution qu'est l'école française, en Kabylie, pendant la colonisation. Faut-il être « roi » pour ne pas le lire. 


\section{BIBLIOGRAPHIE}

BOUALIT Farida, «L'écriture de l'insinuation et du trompe-l'œil de Mouloud Feraoun », Synergies Algérie, N 13, juin-juillet 2011, pp. 19-30.

BOUALIT Farida, « Mouloud Feraoun : l'itinéraire d'un enfant du bled », Djazaïr 2003, Commissariat de l'Année de l'Algérie, n³, Octobre-Novembre, 2003, pp. 4 -7.

BOURDIEU Pierre, «Les rites comme actes d'institution », Actes de la recherche en sciences sociales, Année 1982, volume 43, numéro 43, pp. 58-63. Disponible sur le site : www.persee.fr/web/.../ arss_0335-5322_1982_num_43_1_2159

CNOCKAERT V., PRIVAT J-M, SCARPA M., L'Ethnocritique de la littérature, P.U. Québec, 2011.

De CERTEAU Michel, Invention du quotidien, Tome1, « L'art du quotidien », Gallimard, 1990.

FERAOUN Mouloud, Fils du pauvre, Seuil, 1954.

FERAOUN Mouloud, L'Anniversaire, Seuil, 1972 - Bouchène, 1990.

FERAOUN Mouloud, Lettres à ses amis, ENAG, 1998.

GENETTE Gérard, Discours du Récit, Seuil, 2007.

MATHIEU-JOB Martine et ELBAZ Robert, Mouloud Feraoun ou l'émergence d'une littérature, Karthala, 2001.

PRIVAT J.-M., SCARPA M. éds., Horizons ethnocritiques, Presses Universitaires de Nancy, collection Ethnocritiques, 2010.

SCARPA Marie, Le Carnaval des Halles, Etude sociocritique du Ventre de Paris de Zola, CNRS Editions, 2000.

SEGALEN Martine, Rites et rituels contemporains, Nathan, 1998.

VAN GENNEP Arrnold, Les Rites de passage. Etude systématique des rites, Picard, 1981, (1 ${ }^{\text {ère }}$ ed.1909).

\section{NOTES}

1. D'après Arnold Van Gennep, tous les rites de passage sont composés selon une structure ternaire : une "phase de séparation» (ou préliminaire), suivie d'une "phase de marge " (ou liminaire), parachevée par une "phase d'agrégation» (ou postliminaire) qui marque un changement de statut. Cf. Van Gennep Arnold, Les Rites de passage. Etude systématique des rites, Picard, 1981 (1 $1^{\text {ère }}$ ed.1909), pp. 14 et 27.

2. P. Bourdieu propose de dépasser le stade de la description des rites de passage en se posant la question de la «fonction sociale du rituel et de la signification sociale de la ligne, de la limite, dont le rituel licite le passage, la transgression ». C'est la raison pour laquelle il opte pour la notion de « rite d'institution »: " Parler de rite d'institution, c'est indiquer que tout rite tend à consacrer ou à légitimer, c'est-à-dire faire méconnaître en tant qu'arbitraire et reconnaître en tant que légitime, naturelle, une limite arbitraire.». Cf. "Les rites comme actes d'institution »-Actes de la recherche en sciences sociales, Année 1982, volume 43, numéro 43, pp. 58-63. Disponible sur le site: www.persee.fr/web/.../ arss_0335-5322_1982_num_43_1_2159

3. Cette formule est souvent utilisée par les fondateurs de l'ethnocritique. On la retrouve, par exemple, dans l'ouvrage de Marie Scarpa, L'Eternelle jeune fille. Lecture ethnocritique du Rêve de Zola 
(Honoré Champion, 2009), et dans l'ouvrage collectif de V. Cnockaert, J.-M. Privat, M. Scarpa, L'Ethnocritique de la littérature (P.U. Québec, 2011).

4. Article consulté sur le site www.ethnocritique.com/wa.../pour_une_lecture_ethnocritique.pdf, en décembre 2012.

5. La citation de Mouloud Feraoun est extraite de son article «La littérature algérienne », publié en 1957, dans Revue française et repris dans L'Anniversaire (Bouchène, pp. 59-65).

6. Synergies Algérie $\mathrm{N}^{\circ} 13,2011$, pp. 19-30.

7. Les arguments pour conforter cette thèse n'ont pas manqué. Citons le premier, à savoir le discours prononcé, le 5 avril 1952, par M. Feraoun, à la remise du Prix de la Ville d'Alger, et dans lequel la critique a retenu qu'il s'était défini lui-même comme le "produit authentique » de «l'école française d'Algérie ». Pour prouver le bien-fondé de cette déclaration au niveau littéraire, certains lecteurs inspirés par les travaux de Renée Balibar (Les Français fictifs, Hachette, 1974), se sont appliqués à lire au premier degré les énoncés des manuels scolaires de l'école française repérés dans des textes comme Le Fils du pauvre.

8. Cet article a été publié dans Djazaïr $\mathrm{N}^{\circ}$ 3, Commissariat de l'Année de l'Algérie, 2003.

\section{RÉSUMÉS}

Dans cette brève étude, notre objectif est de démontrer que la "thématisation » de l'école française, dans le contexte de la société kabyle pendant la colonisation, est pour le moins ambivalente dans Le Fils du pauvre de M. Feraoun. Du point de vue de sa représentation en tant que fait culturel, l'école française est bien décrite comme un « rite de passage » (en soi), selon la conception d'A. Van Gennep. Mais ce rite, tel que vécu par l'initié Fouroulou, en chacune de ses trois étapes, n'est pas accompli dans sa plénitude parce qu'il n'est pas soutenu par l'acte de foi sur lequel repose tout rite de passage. Pour saisir le sens de cette attitude de Fouroulou Menrad/ Mouloud Feraoun, nous avons convoqué la notion de " rite d'institution », forgée par P. Bourdieu pour mettre l'accent sur la fonction sociale du rite de passage. Nous concluons sur le peu crédit à accorder à la thèse de "Mouloud Feraoun assimilationniste", "fervent adepte de l'école française ».

In this brief study, we want to analyze the role of the colonial French school in the novel of Mouloud Feraoun "The Son of the Poor Man" (Le Fils du pauvre). The question is important because certain critics assert that this autobiographical novel reveals that the writer is a fervent follower of the French colonial school thus of his ideology. It would mean that Mouloud Feraoun would have successfully undergone the (school) "rite of passage" in its three stages, according to the definition of A.Van Gennep. The hypothesis we shall try to demonstrate in this article is that Fouroulou Menrad/Mouloud Feraoun undergoes actually the "rite of passage" but as "rite of institution", according to the definition of P. Bourdieu. This allows us to show that without failing in the rite of passage, Fouroulou Menrad/Mouloud Feraoun refuses to accept to submit himself to it. The notion of rite of institution reveals the condition of the rite which this writer does not accept: overtake the limit which separates him from his people. Our conclusion thus contradicts the thesis according to which Mouloud Feraoun would be the defender of the colonial French school. 
INDEX

Mots-clés : rite de passage, rite d'institution, ethnocritique, Mouloud Feraoun, Le Fils du pauvre Keywords : rite of passage, rite of institution, ethnocriticism, Mouloud Feraoun, The Son of the poor man

\section{AUTEUR}

\section{FARIDA BOUALIT}

Université A. Mira - LAILEMM - Bejaia 